Estudios Geográficos, LXIX, 265, pp. 519-543, julio-diciembre 2008

ISSN: 0014-1496

elSSN: $1988-8546$

doi: 10.3989/estgeogr.0427

\title{
Ecología del Paisaje. Un marco para el estudio integrado de la dinámica territorial y su incidencia en la vida silvestre
}

\section{Landscape Ecology. A framework for the integrated study of landscape dynamics and its incident in wildlife}

\author{
Mikel Gurrutxaga San Vicente* \\ $\mathrm{y}$ \\ Pedro J. Lozano Valencia ${ }^{\star \star}$
}

\section{Bases disciplinares de la Ecología del Paisaje}

La Ecología del Paisaje estudia los patrones espaciales y estructurales del territorio teniendo en cuenta los procesos y flujos que tienen lugar en el mismo. Como punto de partida asume que la heterogeneidad espacio-temporal del paisaje, resultante de la interacción dinámica de las sociedades humanas con el medio, controla diversos movimientos y flujos de organismos, materia y energía (Forman y Godron, 1986; Noss, 1991; Pickett y Cadenasso, 1995).

La Ecología del Paisaje entiende éste como la manifestación en el espacio de la interacción dinámica entre las sociedades humanas y el medio (Bertrand, 1975), formado por un mosaico espacial heterogéneo de teselas con diferentes características (Forman y Godron, 1985). Existe como estructura y sistema ecológico, independientemente de la percepción (Bertrand, 1975). Así, en el marco de la Ecología del Paisaje, éste constituye un nivel de organización de los sistemas ecológicos, por encima del ecosistema y por debajo de la ecorregión (Forman, 1995; Burel y Baudry, 1999). En un

* Departamento de Medio Natural y SIG. IKT, S.A. (mgurrutxaga@ikt.es).

** Departamento de Geografía, Prehistoria y Arqueología, UPV-EHU (fgplovap@vc.ehu.es). 
nivel superior de organización, la ecorregión constituye un territorio en el que se da una característica combinación de paisajes (Forman, 1995).

Si bien su marco conceptual y metodológico se ha venido gestando durante varias décadas, la Ecología del Paisaje no ha adquirido la importancia de una disciplina científica diferenciada hasta tiempos relativamente recientes (Forman, 1995). Hasta los años 1970 esta aproximación estuvo fuertemente dominada por los geógrafos, vinculada al estudio de las potencialidades ecológicas de vastos territorios. Los geógrafos del este de Europa, con larga tradición en planificación, fueron los más prolíficos durante esa época (Burel y Baudry, 1999). A partir de los años setenta comenzaron a emerger con fuerza estudios realizados por ecólogos sobre las consecuencias de la fragmentación forestal en Norteamérica. En 1982 se creó la asociación científica internacional de Ecología del Paisaje, International Association for Landscape Ecology (IALE).

El término Ecología del Paisaje fue introducido en 1939 por el geógrafo alemán Carl Troll, cuatro años después de que el botánico inglés Tansley (1935) acuñara el de ecosistema, con el objetivo de integrar la componente espacial en la ecología de sistemas. La Ecología del Paisaje es tributaria, en cierta medida, de la Ciencia del Paisaje, cuyo precedente hay que situarlo en el autor Vidal de La Blache y posteriores geógrafos como Schlütter y Max Sorre, hasta llegar a la configuración de la Escuela Francesa de Geografía, que considera el paisaje como el objeto de estudio y estructuración de la Geografía Física. En este contexto habría que reseñar autores como Tricart, pero fundamentalmente Bertrand, autor de una metodología ya concreta y seguida durante décadas, no sólo por la Geografía francesa, sino también por la española (Martínez de Pisón, 1983; Bolós, 1992).

La concepción de la Ecología del Paisaje sobre éste coincide con la consideración del territorio como un tejido vivo a modo de trama, en la que los diferentes componentes, físicos y biológicos, se encuentran relacionados y conectados mediante procesos de interacción (Díaz Pineda y Schmitz, 2003). Paralelamente, se parte de la base de que en los sistemas ecológicos se producen procesos a diferentes escalas espacio-temporales (Turner et al., 1989). Así, como sujetos dinámicos, los sistemas ecológicos incluidos en la corteza terrestre están sometidos a cambios ambientales de escala espacial y temporal muy variable. La dinámica climática del planeta y los regímenes naturales de perturbación no pueden explicar por sí solos la evolución de los ecosistemas. Así, la incidencia de las actividades antrópicas como poderosos agentes de modificación, transformación y creación de ecosistemas y paisajes ha adquirido una importancia crucial en el estudio de los espacios 
humanizados (Zonneveld, 1995). Ello viene a contradecir o, como mínimo, matizar considerablemente, la escasa importancia que estudios, investigaciones y tradiciones científicas han venido otorgando al peso que las actividades del ser humano han tenido en el devenir y desarrollo de los distintos ecosistemas.

La Ecología del Paisaje se caracteriza por estudiar el territorio, a diferentes escalas espaciales, de forma integrada y con de un enfoque sistémico. En todo sistema o conjunto de elementos relacionados, las variaciones en las características de un elemento modifican al conjunto. Por ello, en el análisis del sistema resulta crítico considerar las relaciones existentes entre los elementos. En la medida en que la estructura de un sistema está formada tanto por las características de los elementos, cuya modificación es dificultosa, como por las relaciones entre dichos elementos, éstas destacan como responsables esenciales de la dinámica del sistema, y por tanto de toda problemática del mismo que se pretenda analizar y corregir. En esta línea, la Ecología del Paisaje se caracteriza por dar mayor relevancia a los procesos que, en la escala espacial y temporal, relacionan a los factores (vegetación, fauna, suelo, agua, actividades antrópicas, etc.) y componentes (unidades espaciales) del paisaje, que a las características intrínsecas de los factores y componentes mismos. De esta manera, para la completa comprensión del funcionamiento del paisaje es necesario, además de identificar los procesos de interacción entre los elementos, considerar las escalas espacio-temporales a las que se manifiestan y sus relaciones de dependencia jerárquica (Zonneveld, 1995; Montes, 1998; Burel y Baudry, 1999; De Lucio et al., 2003).

Por consiguiente, las interacciones que determinan el funcionamiento ecológico del sistema territorial son responsables esenciales de la estructura de los paisajes y su dinámica. Recíprocamente, la estructura paisajística resultante, caracterizada por su heterogeneidad espacio-temporal, actúa controlando diversos procesos funcionales que tienen lugar en su seno, como son los distintos flujos de organismos, materia y energía.

Bajo la idea básica de que la Ecología del Paisaje analiza los patrones paisajísticos como resultado de la interacción dinámica entre las actividades antrópicas y la naturaleza, dicha disciplina, trata de estudiar, más concretamente, tanto la manera en que las actividades antrópicas modifican los elementos que conforman el paisaje, sus características y sus relaciones espacio-funcionales, como la forma en que los organismos silvestres y los ciclos geoquímicos se comportan ante la calidad y disposición de dichos elementos. 
La importancia de las investigaciones encaminadas a la comprensión de las implicaciones funcionales de la dinámica paisajística radica en su aplicación por parte de los planificadores y gestores del territorio en la toma de decisiones, sobre objetivos como el mantenimiento de la biodiversidad, la gestión sostenible de los recursos naturales y la planificación territorial con base ecológica (Boyce, 1995; Dramstad et al., 1996; Montes, 1998; Opdam et al., 2002; Ryszkowski, 2002; Díaz Pineda y Schmitz, 2003; De Lucio et al., 2003; Irastorza, 2006).

Para estudiar los patrones estructurales del paisaje, es decir, la disposición y características espaciales de los elementos que lo conforman, la Ecología del Paisaje trata de establecer métodos objetivos para cuantificarlos y convertirlos en medidas cartográficas (Turner et al., 2001; Vila et al., 2006). Esta labor está siendo facilitada por el uso de la tecnología de los Sistemas de Información Geográfica, los cuales sirven de soporte a diferentes programas diseñados para calcular los atributos espaciales del paisaje y sus componentes. El análisis de los patrones del paisaje permite comparar desde un punto de vista estructural diferentes áreas de estudio, o un mismo sector en momentos diferentes (Romero, 2005).

Si bien los patrones espaciales sirven para describir la estructura del paisaje, para establecer relaciones entre ésta y los aspectos funcionales se han de dotar de sentido ecológico a las medidas obtenidas (Vos et al., 2001). Para ello es preciso comprobar la relación entre la estructura del paisaje y los procesos ecológicos objeto de estudio, o entre la estructura paisajística y ciertos parámetros que sean reflejo de dichos procesos. Dicha comprobación ha de realizarse mediante estudios empíricos (Atauri y de Lucio, 2001). Otra aproximación consiste en modelizar dicha relación, en base a una hipótesis previa, para después testar la modelización realizada mediante estudios empíricos (Gurrutxaga, 2007).

\section{LAS RELACIONES ENTRE LA ESTRUCTURA DEL PAISAJE Y LA VIDA SILVESTRE: CONCEPTOS Y APLICACIONES}

Uno de los principales objetos de estudio de la Ecología del Paisaje es el conjunto de relaciones que se establecen entre la estructura y composición del mismo y los procesos ecológicos que regulan la dinámica de poblaciones y la composición de las comunidades biológicas. Este ámbito temático nos permite desarrollar importantes bases conceptuales y aplicaciones dentro de la mencionada disciplina científica. Así, a continuación 
se describen las bases conceptuales fundamentales al respecto, para después tratar las aplicaciones de esta temática en la planificación y gestión territorial.

\section{Estructura del paisaje}

La Ecología del Paisaje adopta un modelo estructural de éste formado por tres tipos de elementos básicos (Forman y Godron, 1981 y 1986). La matriz constituye el elemento espacial dominante y englobante en el que se insertan el resto de los elementos paisajísticos. Las manchas son aquellos elementos espaciales no lineales insertos en la matriz, con características propias y bien diferenciadas de la misma. Por último, los corredores son los elementos lineales, que pueden aparecer en el paisaje aislados o bien conectando otros elementos (manchas) entre sí (figura 1).

\section{Figura 1. MODELO ESTRUCTURAL DEL PAISAJE MATRIZ-MANCHA CORREDOR}

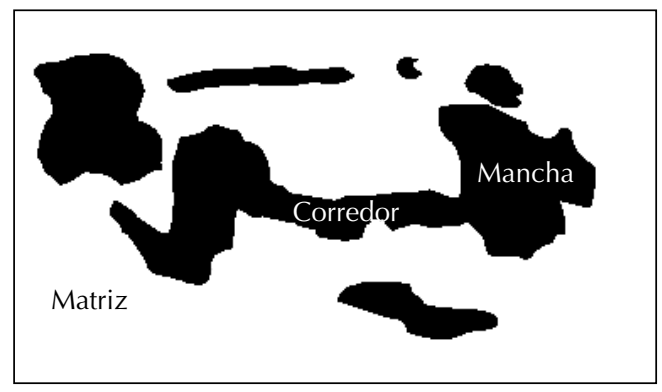

Fuente: Forman y Godron (1986).

El paisaje se define desde el punto de vista estructural como el mosaico espacial formado por teselas, cada una de las cuales conforma un ecotopo diferenciado (Forman, 1995). Un ecotopo corresponde a la unidad ecológica elemental definida espacialmente, es decir, la unidad homogénea mínima de paisaje cartografiable que representa la ubicación espacial de una unidad con características propias.

La representación de la estructura del paisaje depende de los objetivos de estudio y de la escala espacial de análisis, motivo por el cual la estructura del mismo sector del espacio geográfico puede ser representada de diferentes maneras. Así, al considerar las especies como sujetos relacionados con la estructura del paisaje, es preciso integrar el concepto de hábitat, que se corresponde con aquel área que reúne las condiciones y recursos necesarios 
para ser ocupada por un organismo dado, donde desarrolla su vida y se reproduce (Hall et al., 1997). La relación entre hábitat y ecotopo varía en función del taxón considerado y del teselado adoptado, pudiendo estar un hábitat conformado por diferentes ecotopos. Esto enlaza con el concepto de unidad funcional, definido por Merriam (1984) como el conjunto de elementos paisajísticos utilizados por una especie en su ciclo de vida. Cuando un ecotopo o tesela se corresponde con un hábitat, se le denomina también mancha o fragmento de hábitat.

\section{Escala espacial}

Un aspecto de gran importancia a tener en cuenta es que el patrón estructural de un paisaje varía en función de la escala espacial de análisis (Turner et al., 1989). Ésta presenta dos componentes: el ámbito espacial de estudio, o extensión, y el tamaño mínimo de los elementos del paisaje o teselas que se incluyen en el análisis (resolución o tamaño de grano). Si bien el concepto de escala pequeña y grande posee significados antagónicos según diferentes autores, más comúnmente se identifican ámbitos de estudios pequeños y grados de resolución detallados con escalas de análisis pequeñas, y ámbitos grandes y grados de resolución gruesos con escalas grandes (Forman y Godron, 1986; Antúnez y Márquez, 1992).

La escala de representación de la estructura del paisaje debe adecuarse al objeto de estudio, es decir, debe ser la adecuada para detectar aqueIlas relaciones que se deseen analizar entre la estructura del paisaje y los aspectos funcionales que en él tengan lugar.

En primer término, a este respecto de las escalas de representación del paisaje, hay que señalar que, en razón del propio origen de la disciplina como confluencia de ciencias naturales y sociales, la Ecología del Paisaje considera a éste como un espacio concerniente a las actividades humanas. Así, la escala espacial a la que esta disciplina estudia los patrones paisajísticos corresponde fundamentalmente a lo que se ha venido a denominar la "escala de paisaje", que trasladada al territorio abarca comúnmente desde algunas hectáreas hasta algunos cientos de km² (Burel y Baudry, 1999). De esta forma, los conceptos de paisaje y ecotopo o tesela implican una escala de observación que coincide, en términos generales, con el concepto intuitivo/perceptivo humano de paisaje (de Lucio et al., 2003). Esta "escala de paisaje" es considerada la idónea para la toma de decisiones referentes a la planificación y gestión territorial (Burel y Baudry, 1999; Europarc-España, 2002; De Lucio et al., 2003; García Fernández-Velilla, 2003). 
No obstante, para el estudio de las relaciones entre la estructura del paisaje y la dinámica de poblaciones silvestres es preciso considerar la escala de percepción del paisaje del taxón en cuestión, a la que éste responde a dicha estructura (With y Crist, 1995; Tischendorf y Fahrig, 2000; SuárezSeoane y Baudry, 2002). Así, lo que para una especie puede constituir una parcela homogénea, para otra puede tratarse de un medio muy heterogéneo. Por ello, es necesario conocer en detalle la ecología espacial de las especies para adoptar una adecuada representación de la estructura del paisaje en cada caso. De esta forma, la escala de percepción de los organismos de la estructura del paisaje se corresponde con aquella en la que perciben su heterogeneidad espacial.

\section{Heterogeneidad}

La heterogeneidad estructural del paisaje viene marcada por la diversidad de elementos paisajísticos que contiene, así como por la complejidad de las relaciones espaciales entre éstos (Forman y Godron, 1986; Forman, 1995; Burel y Baudry, 1999).

La teoría de la heterogeneidad del hábitat predice que una mayor heterogeneidad del paisaje se asocia con una mayor biodiversidad, al existir una mayor diversidad de tipos de hábitats (Williams, 1964). En este sentido, diversos estudios han mostrado la relación positiva entre la heterogeneidad de los mosaicos agrarios y la riqueza de especies asociadas (Pino et al., 2000; Atauri y de Lucio, 2001; Benton et al., 2003; Farina, 2004). Así, se ha comprobado que los procesos de homogeneización de los paisajes agrarios acontecidos en Europa, como consecuencia de la intensificación de los aprovechamientos, ha provocado la pérdida de numerosas especies asociadas a los agrosistemas tradicionales (Tucker, 1997; Maes y Van Dyck, 2001; Donald, 2004). También el abandono de la actividad agrícola en áreas menos productivas causa, en numerosos casos, la disminución de la diversidad biológica asociada al territorio (González Bernáldez, 1991; SúarezSeoane et al., 2002), al existir una numerosa comunidad de organismos adaptados a los agrosistemas estructuralmente heterogéneos.

No obstante, niveles altos de heterogeneidad pueden estar asociados a un elevado grado de fragmentación de hábitats y a la consiguiente presencia de manchas muy pequeñas incapaces de albergar poblaciones viables de ciertos organismos. Así, el incremento de la diversidad asociado a la heterogeneidad puede presentar un valor máximo por encima del cual empiece a disminuir (Santos y Tellería, 1998). 


\section{Fragmentación}

Desde un punto de vista espacial, los procesos de fragmentación de hábitats pueden definirse genéricamente como la división de una o varias manchas de hábitat original en una serie de fragmentos que suman una superficie total menor a la inicial.

La fragmentación del paisaje está estrechamente relacionada con el tamaño de las manchas de hábitat presentes en el mismo. Aunque, en suma, la superficie total de hábitat disponible sea la misma, un paisaje está más fragmentado cuanto menor es el tamaño de las manchas de hábitat y por consiguiente el número de fragmentos es mayor. Asimismo, el grado de separación entre los fragmentos de hábitat se integra dentro del concepto de fragmentación espacial, en oposición al término de conectividad espacial, que se refiere a la contigüidad o adyacencia de las manchas de hábitat en el espacio.

La alteración de los patrones espaciales de las manchas de hábitat, derivada de los procesos de fragmentación, se manifiesta a través de ciertas tendencias fundamentales (Saunders et al., 1991; Andrén, 1994; Fahrig, 2003), tal y como puede observarse en la figura 2:

a) disminución de la superficie total de hábitat,

b) disminución del tamaño de los fragmentos,

c) aumento del número de fragmentos,

d) aumento de la separación entre los fragmentos,

e) aumento de la relación perímetro/superficie de los fragmentos.

Figura 2. ESTADOS DE CRECIENTE FRAGMENTACIÓN DEL HÁBITAT

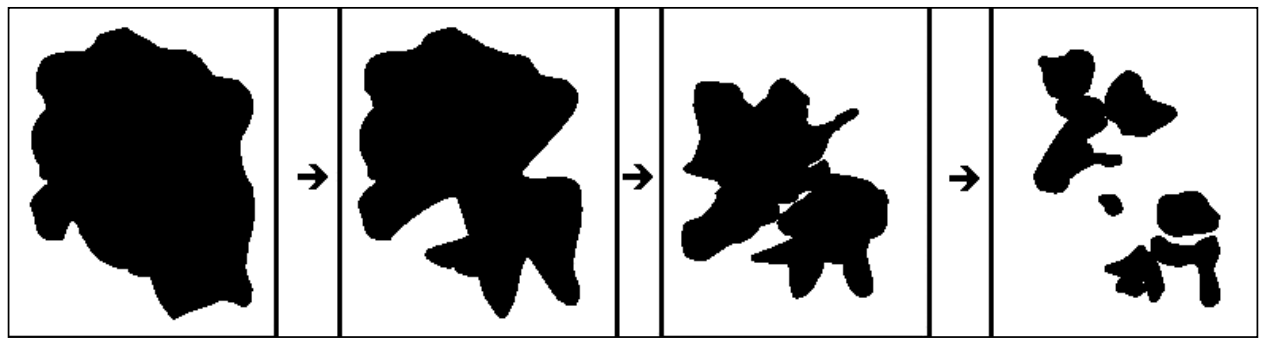

Fuente: Modificado de Bennett (1999).

Otra aproximación al concepto de fragmentación en relación a las especies se refiere a la dinámica de la distribución espacial de éstas, de forma que las mismas tendencias apuntadas para la fragmentación de hábitats pueden 
afectar a las áreas ocupadas por los núcleos de población de los taxones. Conceptualmente, la diferencia más importante entre ambos procesos estribaría en que, en el caso de los organismos, además de que éstos sólo ocupan parte de los territorios donde podrían encontrarse (Antúnez y Mendoza, 1992), al aumentar la fragmentación se producirían fenómenos de extinción local, al formarse fragmentos demasiado pequeños para ser ocupados por la especie, de manera que lo que puede ser un fragmento de hábitat adecuado no siempre corresponde a un núcleo de población ni al área de distribución

Esto enlaza con conceptos de componente espacial referidos a la dinámica de poblaciones, como el área mínima viable (MVA, minimum viable area) (Shaffer, 1987), que se refiere a la superficie mínima de hábitat aislado que necesita la población para sobrevivir a largo plazo.

En cambio, cuando los fragmentos no están aislados para la especie y existe intercambio de individuos, las manchas no constituyen diferentes poblaciones. Esto enlaza con el concepto de metapoblación, propuesto por Levins (1970) como una población formada por un conjunto de poblaciones locales, distribuidas en diferentes parches, que se extinguen y recolonizan localmente.

Como proceso fundamentalmente antropogénico, causado por la incidencia de ciertas actividades humanas sobre el territorio, los procesos de fragmentación de hábitats modifican los patrones espaciales y la configuración del paisaje, pudiendo alterar no sólo la distribución de los organismos, sino la integridad de ciertos procesos ecológicos. La fragmentación de hábitats se asocia fundamentalmente a rápidos y severos cambios en los usos del suelo acontecidos globalmente a partir del siglo XX, favorecidos por el gran aumento demográfico de la población humana y por el desarrollo industrial y tecnológico. De entre el complejo conjunto de flujos y procesos que pueden verse afectados por la fragmentación de hábitats, son los de desplazamiento, dispersión y migración de los organismos silvestres a través del territorio uno de los más estudiados, así como los flujos de intercambio genético entre poblaciones, estrechamente relacionados con los anteriores (Vandewoestijne y Baguette, 2004). Esto enlaza con el concepto de conectividad funcional, que se desarrolla a continuación.

\section{Conectividad espacial y funcional}

Como se ha indicado anteriormente, la conectividad espacial se refiere a la contigüidad o adyacencia de las manchas de hábitat en el espacio. Así, cuanto más separados o distanciados estén los fragmentos de hábitat entre ellos, menor conectividad espacial tendrá dicho hábitat en el paisaje. 
Sin embargo, la conectividad es descriptor de las relaciones entre las machas tanto desde el punto de vista espacial como funcional.

Así, el concepto de conectividad funcional se refiere a la capacidad del territorio para permitir los desplazamientos de los organismos entre las teselas o ecotopos con recursos (Taylor et al., 1993). Análogamente, la conectividad funcional se define asimismo como la capacidad de un individuo o sus formas de propagación para desplazarse entre manchas, independientemente de la distancia que separe éstas (Baudry y Merriam, 1998).

Por tanto, la conectividad funcional es una propiedad del territorio para una especie determinada o para un conjunto de especies con similares requerimientos ecológicos y capacidad dispersiva (Del Barrio et al., 2000). De esta forma, especies similares en su grado de movilidad, perfil ecológico y modo de percibir la estructura del paisaje, conforman un grupo funcional, en relación al cual puede estudiarse, desde un enfoque integrado multiespecífico, la conectividad funcional del paisaje (Gurrutxaga, 2007). En cuanto al concepto de permeabilidad, que en ocasiones se emplea como sinónimo al de conectividad, se refiere a una propiedad más general del paisaje referida al mantenimiento de la conectividad para el conjunto de las diferentes especies que lo habitan (De Lucio et al., 2003). Este mismo concepto suele emplearse para referirse al grado de permeación de organismos que una determinada barrera lineal, normalmente antrópica (carreteras, vías férreas, canales y conducciones hídricas, etc.) puede ejercer (Yanes et al., 1995).

La relación entre la conectividad espacial y la conectividad funcional en el paisaje no siempre es estrecha. Los medios de desplazamiento y dispersión de ciertas especies permiten que, incluso en paisajes donde el hábitat de éstas se distribuya en escasas manchas muy distanciadas (conectividad espacial muy baja), la conectividad funcional sea elevada. Este es el caso de ciertas especies vegetales cuyas semillas se dispersan por el viento (anemocoria), así como de ciertas especies de invertebrados y aves que se desplazan a grandes distancias volando. En cambio, en el otro extremo se encuentran ciertos taxones con escasa movilidad y alta especialización de hábitat, para los cuales la relación entre la conectividad espacial y funcional es muy estrecha. Por ejemplo, insectos saproxílicos como Osmoderma eremita dependen de la presencia de rodales de árboles viejos situados a escasa distancia, que presenten oquedades con madera en descomposición, para mantener poblaciones. Éstas quedan aisladas y tienden a la extinción si no existen otros rodales cercanos, dado que la capacidad dispersiva de dicha especie se reduce a varios centenares de metros (Ranius y Hedin, 2001). 
En la figura 3 se exponen, de forma esquemática, diferentes relaciones que pueden presentar la conectividad espacial y funcional, dependiendo de las características del paisaje y de la especie en cuestión. Es preciso señalar que en dicha figura se adopta una aproximación simplificada, ya que se toma un modelo de paisaje binario, compuesto por manchas de hábitat y matriz. Sin embargo, las características de los diferentes ecotopos que forman la matriz en la que se insertan las manchas de hábitat presentan un papel fundamental en la regulación de los flujos bióticos que tienen lugar entre éstas. Además, como se ha señalado anteriormente, el hábitat de una especie puede estar formado por diferentes tipos de ecotopos. Así, en los estudios dirigidos a encontrar las relaciones entre la estructura del paisaje y la movilidad de los organismos se requiere la adecuada plasmación de la heterogeneidad del paisaje, tal y como la perciben éstos.

Figura 3. RELACIÓN ENTRE CONECTIVIDAD ESPACIAL Y FUNCIONAL EN EL PAISAJE, EN FUNCIÓN DE LA ECOLOGÍA ESPACIAL DE LAS ESPECIES

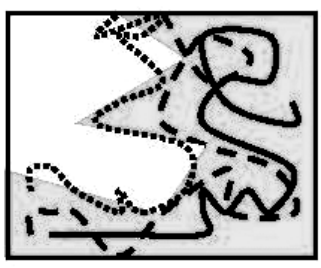

a) conectividad espacial alta

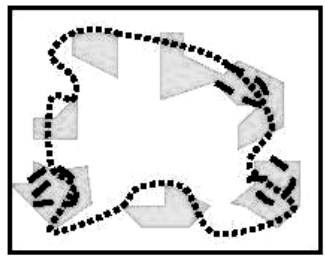

c) conectividad espacial baja

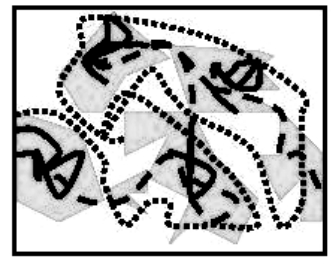

b) conectividad espacial media

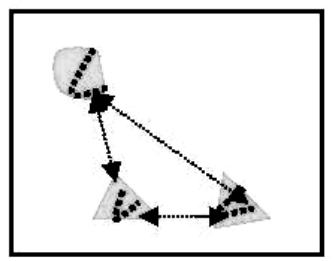

b) conectividad espacial muy baja

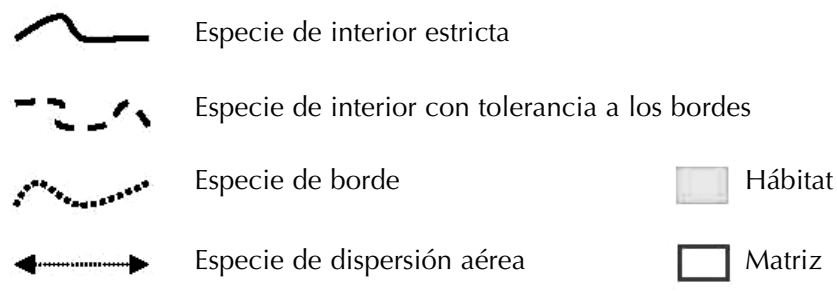

Fuente: Modificado de Burel y Baudry (1999). 
En la situación a) de la figura 3 la conectividad espacial del hábitat es elevada, al existir una mancha de amplia extensión, de forma que tanto para las especies de interior — su hábitat se encuentra en la parte interna de los fragmentos y no toleran en la misma medida las zonas de borde con otros ecotopos - como para las especies de borde - tolerantes a las zonas de ecotono - el paisaje es permeable a sus movimientos y la conectividad funcional es alta.

En la situación b) la conectividad espacial del hábitat es menor, de forma que para las especies estrictamente de interior la conectividad funcional se reduce de forma severa, mientras para las especies de interior menos sensibles a la fragmentación que las anteriores, la conectividad funcional sigue siendo elevada, así como para las especies de borde, que ven ampliado su hábitat.

En la c) la fragmentación del hábitat es elevada y la conectividad espacial baja, impidiendo la presencia de especies estrictamente de interior que requieren fragmentos de hábitat de cierto tamaño. La conectividad funcional para las especies de interior menos sensibles a la fragmentación se reduce drásticamente, al no poder desplazarse grandes distancias a través de la matriz. Para las especies de borde el paisaje sigue presentando una conectividad funcional elevada, ya que disponen de gran superficie de bordes y la matriz no supone una barrera infranqueable a sus movimientos.

En la d) la conectividad espacial del hábitat es muy baja y los fragmentos muy pequeños, de forma que desaparecen las especies de interior. La conectividad funcional para las especies de borde pasa a ser muy reducida y sus poblaciones van quedando aisladas debido a que ha aumentado notablemente la distancia entre los bordes de los fragmentos, constituyendo ahora sí la matriz una barrera por su extensión. Tan sólo las especies ubiquistas y aquellas que no lo son pero que poseen medios de dispersión aéreos a larga distancia, pueden desplazarse de un fragmento a otro.

La relación entre conectividad espacial y funcional se relaciona, a su vez, con la vulnerabilidad de los taxones a la fragmentación del hábitat, de forma que los organismos más sensibles se corresponden con los que requieren grandes extensiones de hábitat — cuestión de superficie de hábitat-, y con los que presentan una elevada especialización de hábitat y una limitada capacidad dispersiva — cuestión de distancia entre teselas con recursos-.

Por otro lado, la conectividad funcional está estrechamente asociada al concepto de corredor ecológico. El término corredor fue utilizado por primera vez en un sentido ecológico en el marco de la Biogeografía 
Histórica, para referirse a las rutas preferenciales de dispersión de la biota entre regiones y continentes (Simpson, 1936). Dentro de la Ecología del Paisaje, el concepto de corredor concebido por su función conductora, como lugar preferencial para el paso de organismos que se desplazan entre teselas con recursos, ha ido evolucionando. En un principio los modelos estructurales del paisaje concebían a la matriz como un elemento hostil. En este contexto, los corredores se planteaban como estructuras lineales de hábitat que ponían en contacto manchas de hábitat afín. Así, las primeras propuestas derivadas de los estudios de fragmentación de hábitats argumentaban que los fragmentos que están unidos por corredores de un hábitat similar, tienen un mayor valor para la conservación que los fragmentos de igual tamaño que se encuentran aislados. Estos primeros modelos evolucionaron, incorporándose la heterogeneidad de la matriz y el importante papel de ésta en la regulación de la conectividad ecológica entre las manchas.

De esta forma, actualmente se asume que los corredores ecológicos no se restringen a elementos lineales, sino que constituyen sectores de alta conectividad funcional de la matriz territorial (Del Barrio et al., 2000). Se evita así adoptar un enfoque reduccionista que base el mantenimiento de los flujos biológicos a través de paisajes fragmentados en los elementos paisajísticos lineales y continuos, es decir, en corredores estructurales del modelo matriz-mancha-corredor. De esta forma, la tendencia, que se ha tenido en ocasiones, a confundir forma y función en el concepto de corredor (Rosenberg, 1997), ha sido superada reconociendo el papel fundamental de la heterogeneidad del paisaje en la dinámica de poblaciones.

\section{Aplicaciones en la planificación territorial}

Dentro de las principales tendencias que se han dado en la dinámica de los paisajes europeos durante las últimas décadas, se encuentran la reducción y fragmentación de hábitats naturales y la homogeneización de los paisajes agrícolas (Jongman, 2002). Ambos procesos, causados fundamentalmente por las redes de infraestructuras, la urbanización y la intensificación agraria, son apuntados como una de las principales causas de la actual crisis de biodiversidad (Fahrig, 2003; Foley et al., 2005).

Desde los postulados de la Ecología del Paisaje, en las últimas décadas se han realizado numerosos estudios de constatación de las consecuencias de dichos procesos, y se ha incidido en la búsqueda de soluciones para hacer frente a la crisis de biodiversidad. En este marco, los 
enfoques centrados en los patrones del paisaje resultan altamente complementarios con los orientados a especies o grupos funcionales (Fischer y Lindenmayer, 2007).

Una de las líneas de estudio fundamentales se ha dirigido a la constatación de que la intensificación de la agricultura, que ha provocado la disminución de la heterogeneidad del paisaje rural, ha repercutido negativamente sobre el hábitat de una rica comunidad biológica y sobre la conectividad del paisaje para numerosas especies (Burel y Baudry, 2005). Asimismo, se ha señalado la importancia de la matriz agraria como soporte de los futuros cambios en el área de distribución de diversos organismos en respuesta al cambio climático (Hannah et al., 2002, del Barrio et al., 2006). Los efectos negativos de la intensificación agraria tratan de ser mitigados articulando instrumentos como las medidas agroambientales y los procedimientos de certificación de gestión forestal sostenible. No obstante, se constata que aún es insuficiente la integración de los criterios de la Ecología del Paisaje para que la aplicación de estos instrumentos, así como la propia ordenación agraria y forestal, se realice con una perspectiva territorial, y no sólo de parcela o rodal (Pierce y Ervin, 1999; Atienza, 2004; Donald y Evans, 2006).

Paralelamente, se ha puesto de manifiesto con gran consenso que las estrategias de conservación basadas en la protección de espacios naturales concebidos como unidades espacial y funcionalmente independientes no resuelven la necesidad de tomar en consideración los flujos ecológicos que tienen lugar en la globalidad del territorio (Schafer, 1990; Kupfer, 1995). La evidencia de que, dentro de los flujos e interacciones horizontales que tienen lugar en el conjunto territorial, los de desplazamiento e intercambio genético de las poblaciones de fauna y flora silvestres resultan fundamentales para la pervivencia de aquellas especies sensibles a la fragmentación de sus hábitats, ha hecho que evitar la pérdida de conectividad ecológica se configure como una tarea fundamental a afrontar por las políticas de conservación de la biodiversidad (Benett, 1999).

De esta manera, los corredores ecológicos pueden ser concebidos, desde un enfoque como el de la Biología de la Conservación, para favorecer la conectividad entre poblaciones de especies concretas, o entre poblaciones y zonas aptas (Graves et al., 2007). Esta aproximación puede integrarse en las estrategias o planes de conservación de ciertas especies amenazadas, como por ejemplo el oso pardo (Ursus arctos) en la Cordillera Cantábrica (Ministerio de Medio Ambiente, 1999).

Paralelamente, desde un enfoque integrado y de síntesis, los corredores y la conectividad pueden ser concebidos desde una perspectiva de paisaje 
o ecorregional (Hoctor et al., 2000; Fischer y Lindermayer, 2007). Esta aproximación puede aplicarse al diseño de redes ecológicas coherentes, mediante el establecimiento de corredores ecológicos entre espacios naturales protegidos (Gurrutxaga, 2007; ATECMA, 2008). Esto enlaza con el papel cada vez más importante que está tomando la denominada planificación ecorregional en las políticas de conservación de la naturaleza, reconociendo que es preciso integrar ecológica y socioeconómicamente las áreas protegidas en el conjunto del territorio (Bennett y Wit, 2001; Múgica et al., 2002). La aplicación del enfoque ecorregional conlleva desarrollar redes de conservación coherentes y funcionales, denominadas redes ecológicas. Las redes ecológicas se identifican, desde el punto de vista estructural, por la conexión mediante corredores ecológicos de los espacios naturales de mayor relevancia o zonas-núcleo, que actúan como nodos, y por la disposición de zonas de amortiguación entre los elementos anteriores y el resto de la matriz territorial (Bennet y Mulongoy, 2006). El diseño de las redes ecológicas de forma espacialmente explícita permite su implementación en la planificación territorial (Benett, 1999; Vuilleumier y Prelaz-Droux, 2002; Opdam et al., 2006; Huber et al., 2007), afectando a la regulación de los usos del suelo y a los procesos de evaluación de impacto ambiental de planes y proyectos. Este es un proceso aún incipiente, dado que la mayor parte de las iniciativas para desarrollar redes ecológicas en el mundo se encuentra en la fase de planificación y no han sido completamente implementadas (Bennett y Mulongoy, 2006).

El concepto de red ecológica se ha plasmado en la Directiva 92/43/CEE, o Directiva Hábitats que regula el desarrollo de la Red Natura 2000 en la Unión Europea. En su artículo 10 se insta a los estados miembros a mejorar la coherencia ecológica de la red mediante la gestión de aquellos elementos del paisaje que resulten fundamentales para garantizar la migración, distribución geográfica y el intercambio genético de las especies silvestres. Además, la Directiva Hábitats somete a evaluación ambiental a aquellos planes y proyectos que puedan afectar negativamente a la coherencia de la Red Natura 2000. En este sentido, la Directiva 2001/43/CE de evaluación de planes y programas con incidencia en la ordenación del territorio, constituye el instrumento fundamental articulado por la UE para materializar la integración de las redes de áreas protegidas en la práctica de la ordenación del territorio a diferentes escalas espaciales (Mata, 2005).

Dado que la conectividad es una cualidad del paisaje en relación a una especie o grupo funcional y que los beneficiarios de los corredores son 
los organismos silvestres (Taylor et al., 1993; Tischendorf y Fahrig, 2000; Foppen et al., 2000), los métodos de diseño de corredores ecológicos se basan generalmente en especies focales o en grupos funcionales, en función de cuyos requerimientos ecológicos se parametriza y analiza la conectividad del paisaje (Van der Sluis et al., 2004). Los grandes y medianos mamíferos son el grupo zoológico que más se ha utilizado en el diseño de corredores ecológicos a escala regional (Walker y Craighead, 1997; Corsi et al., 2002; Singleton et al., 2002; Bruinderink et al., 2003; J'drzejewski et al., 2005; Gurrutxaga, 2007). Esto obedece a varios factores. En primer lugar, se trata de especies cuyo dominio vital y rangos de desplazamiento son detectables a escala regional. De esta forma, su escala de percepción del paisaje coincide con frecuencia con la de cartografías de vegetación y usos del suelo, con la de percepción humana del paisaje y con la que éste es modelado o gestionado por las actividades antrópicas. Otro criterio es que suelen presentar una distribución, al menos potencialmente, zonal en el área de estudio, de cara a evitar la selección de especies relictas o confinadas a sectores localizados. Además, la transitabilidad de ciertos grandes y medianos mamíferos a través del territorio resulta sensible a los principales procesos dinámicos de los patrones del paisaje causantes de pérdida de conectividad ecológica, como la fragmentación de los bosques naturales, la pérdida de heterogeneidad de los paisajes agrícolas por la intensificación de los aprovechamientos y el efecto-barrera causada por las redes de infraestructuras de transporte. Por último, pueden actuar como especies paraguas (Lambeck 1997), de forma que, si sus requerimientos de hábitat y de transitabilidad son cubiertos en el paisaje, éste tiene capacidad para dar soporte a un amplio grupo de taxones con requerimientos ecológicos análogos o inferiores (Noss y Daly, 2006).

Paralelamente, y a pesar de que constituyen escalas con menor grado de incidencia sobre las redes administrativas de conservación, los corredores ecológicos pueden diseñarse también en función de especies que operan a escalas desde continentales, como aves migratorias, a locales, como anfibios, pequeños mamíferos y ciertos invertebrados. De hecho, las redes ecológicas deben ser multiescalares, de forma que cubran las necesidades de las diferentes especies sensibles a la fragmentación, cada una de las cuales percibe la configuración del paisaje a una determinada escala (Foppen et al., 2000). Esto refuerza el carácter complementario, a la hora de establecer los requerimientos de conectividad en el paisaje, de ambos enfoques, el centrado en taxones concretos y el orientado a integrar los espacios naturales en una red funcional. 


\section{Dimensión TRANSDisciplinar DE LA ECOlOGÍA DEL PaISAJE}

Por último, el ámbito temático de las relaciones que se establecen entre la estructura del paisaje y los procesos ecológicos que regulan la dinámica de poblaciones y la composición de las comunidades biológicas, permite abordar el carácter transdisciplinar de la Ecología del Paisaje.

Así, como suele ocurrir cuando una temática de estudio presenta diversos componentes y facetas, que son objeto de análisis desde diferentes campos y enfoques, se produce una interesante trasgresión de los límites disciplinares. Es en estos casos cuando resulta notorio que las disciplinas científicas presentan numerosos nexos y que, lejos de constituir compartimentos estancos del conocimiento, existen importantes superposiciones e intersecciones entre ellas. Así, la transdisciplinariedad se manifiesta al producirse interpenetraciones entre campos capaces de posibilitar múltiples visiones simultáneas del objeto en estudio.

La Ecología del Paisaje, para obtener una visión completa del objeto de estudio, se nutre de métodos, conceptos y aplicaciones de diferentes disciplinas, de forma que, más allá del sumatorio de los diferentes enfoques, adopta una visión integrada y holística. Para comprender la dimensión transdisciplinar de la Ecología del Paisaje, dentro de la temática de las relaciones que se establecen entre la estructura del paisaje y la dinámica de poblaciones y comunidades biológicas, es preciso tener en cuenta diversos aspectos.

En primer lugar, las variaciones en los patrones estructurales del paisaje afectan tanto a hábitats como a poblaciones y comunidades, de forma que sus consecuencias se manifiestan en los diferentes niveles de organización de la biodiversidad, desde los genes hasta los ecosistemas. De esta manera, los efectos de la dinámica estructural del paisaje sobre la vida silvestre, con especial énfasis en los procesos de fragmentación, son objeto de estudio desde campos como la Biología Molecular, la Ecología de Poblaciones (Autoecología), la Ecología de Comunidades (Sinecología) y la Ecología Funcional o de Sistemas.

Como disciplina que integra los diferentes enfoques citados, cobran una destacada importancia las aportaciones de la Biología de la Conservación, que dirige sus investigaciones a los ecosistemas, comunidades y poblaciones silvestres en peligro. Realiza estudios multidisciplinares (ecología evolutiva, ecología del comportamiento, interacciones bióticas, demografía, dinámica de las poblaciones, especiación, patrones de extinción, flujo génico, etc.) orientadas a proporcionar las bases científicas necesarias 
para la conservación de la diversidad biológica en todas sus formas. En la medida en que la reducción y fragmentación del hábitat conlleva graves problemas para la viabilidad de numerosos taxones, la Biología de la Conservación estudia la dinámica de las poblaciones pequeñas y subdivididas como una de sus principales líneas de investigación.

En relación con las manifestaciones de los efectos de la fragmentación y la pérdida de conectividad funcional sobre la dinámica de las poblaciones a nivel genético, los últimos avances en los métodos moleculares han permitido la aparición del campo de la Genética del Paisaje, Landscape Genetics (Manel et al., 2003), de rápido desarrollo en los últimos años. Se trata de una fusión de la Genética de Poblaciones y la Ecología del Paisaje. Esta disciplina implica la identificación espacial de los parámetros genéticos (aislamiento por distancia, flujo genético etc.) en relación a variables paisajísticas, lo que permite proveerse de información sobre la interacción entre las características del paisaje y los procesos microevolutivos, tales como el flujo génico o la deriva genética.

Asimismo, los cambios en la estructura del paisaje actúan como una causa de variaciones en los patrones de distribución y riqueza de especies en el espacio y en el tiempo, objeto de estudio de la Biogeografía.

En segundo lugar, es preciso destacar la vital importancia de las actividades antrópicas, manifestadas en los usos del suelo del territorio y en las prácticas de manejo de los ecosistemas, como agentes moldeadores de los paisajes humanizados. Ello introduce a diversas Ciencias Sociales (Historia, Geografía, Agronomía) en el estudio de la gestión de los ecosistemas y en el análisis de la evolución de la estructura de los paisajes, entendiendo éstos como el resultado de la interacción de las sociedades humanas con el medio en el que habitan (Burel y Baudry, 1999). Así, y dado que las condiciones de partida y las dinámicas del pasado son determinantes en la comprensión del estado y funcionamiento de los ecosistemas, así como en el estudio de su evolución futura, disciplinas como la Prehistoria y la Arqueología (con sus aplicaciones en la Biogeografía Histórica), por un lado, y la Etnografía, la Antropología, la Geografía Humana y la Agronomía, por otro, se encargan, respectivamente, del estudio de la formación de los paisajes y de las técnicas aplicadas por las sociedades para transformarlos o moldearlos.

En tercer lugar, la transdisciplinariedad de la Ecología del Paisaje se manifiesta en los métodos analíticos de los que se nutre, como la Geoestadística, los Sistemas de Información Geográfica y los modelos matemáticos.

En cuarto y último lugar, la transdisciplinariedad de la Ecología del Paisaje se explica por sus implicaciones en la ordenación territorial, entendida 
como la proyección en el espacio de las políticas económica, social, cultural y ambiental de una sociedad (Gómez Orea, 2002). Ello se debe a que la búsqueda de soluciones a las problemáticas espaciales objeto de estudio debe estar sustentada en criterios a aplicar en la planificación y gestión territorial. No en vano, la ordenación territorial afecta a todas las actuaciones públicas con incidencia territorial (Pujadas y Font, 1998). Así, ciertas políticas verticales como la agrícola, urbanística y de transportes, en interacción con las políticas horizontales ambiental y territorial, constituyen los principales agentes que regulan la estructura y el funcionamiento del paisaje. De ahí que, más allá del campo de las ciencias ambientales y naturales, disciplinas como la agronomía, la ingeniería de obras públicas o la arquitectura urbanística incorporen de forma progresiva métodos aplicados para la toma en consideración de criterios de Ecología del Paisaje en sus planes y proyectos. En este sentido, la integración del conjunto de los criterios ambientales en la ordenación territorial queda recogida en la noción de planificación física con base ecológica (Ramos Fernández, 1979).

\section{CONCLUSIONES}

A modo de conclusión, la Ecología del Paisaje presenta dos componentes fundamentales, uno geográfico, que se ocupa de estudiar los patrones espaciales del paisaje, y otro ecológico, referida a los procesos ecológicos que tienen lugar en éste a diferentes escalas. Adopta un enfoque integrado y sistémico de estudio del territorio, con una dimensión claramente transdisciplinar. Las potencialidades de la Ecología del Paisaje en el estudio de las repercusiones de la dinámica territorial sobre la vida silvestre, así como en el planteamiento de soluciones aplicadas a la conservación de la biodiversidad y a la planificación territorial con base ecológica, confieren a esta disciplina un importante papel en la investigación y en la toma de decisiones. La Geografía aplicada tiene en la Ecología del Paisaje un marco adecuado, susceptible de ser impulsado con fuerza en los próximos años, para orientarlo a la resolución de problemas espaciales y a la planificación territorial (Kent, 2007).

\section{Agradecimientos}

El presente artículo se enmarca dentro del proyecto, financiado por la Dirección de Biodiversidad del Departamento de Medio Ambiente y Ordenación del Territorio del Gobierno Vasco, para el diseño de la red de corredores 
ecológicos de la Comunidad Autónoma del País Vasco, encargado a la empresa IKT, S.A. Agradecemos también la ayuda prestada por el Dr. Gabriel del Barrio, de la Estación Experimental de Zonas Áridas del Consejo Superior de Investigaciones Científicas.

Recibido 02.06.08

Aceptado 25.09.08

\section{BIBLIOGRAFÍA}

Andrén, H. (1994): "Effects of habitat fragmentation on birds and mammals in landscapes with differentes proportions of suitable habitat: a review", Oikos, $n^{\circ} 71$, pp. 355-366.

Antúnez, A. y Márquez, A. L. (1992): "Las escalas en Biogeografía", en Vargas, J. M.; Real, R. y Antúnez, A. (eds.), Objetivos y métodos biogeográficos. Aplicaciones en Herpetología, Monografías de Herpetología, vol. 2, Asociación Herpetológica Española, Valencia, pp. 31-38.

Atauri, J. A. y De Lucio, J. V. (2001): "The role of landscape structure in species richness distribution of birds, amphibians, reptiles and lepidopterons in Mediterranean landscapes", Landscape Ecology, no 16, pp. 147-159.

ATECMA, S.L. (2008): Identificación y diagnóstico de la red de corredores ecológicos de la Región de Murcia, Dirección General del Medio Natural de la Región de Murcia.

Atienza, J. C. (2004): Efectos de la reforestación en zonas agrarias sobre la fauna, Tesis doctoral, Universidad Complutense de Madrid.

Baudry, J. y Merriam, H. G. (1988): "Connectivity and connectedness: functional versus structural patterns in landscapes", en Schreiber, K. F., Connectivity in Landscape Ecology (Edited by Proceedings of the 2nd international seminar of the International Association of Landscape Ecology, Münster, Germany, July 1987), Münstersche Geographische Arbeiten, 29, Münster, pp. 23-28.

Bennett, A. F. (1999): Linkages in the Landscape. The role of corridors and connectivity in wildlife conservation, IUCN, Gland, Switzerland and Cambridge, UK.

Bennett, G. y Mulongoy, K. J. (2006): Review of experience with ecological networks, corridors and buffer zones, Secretariat of the Convention on Biological Diversity, Montreal.

Bennett, G. y Wit, P. (2001): The development and application of ecological networks: a review of proposals, plans and programmes, AIDEnvironment, Ámsterdam.

Benton, T. G.; Vickery, J. A. y Wilson, J. D. (2003): "Farmland biodiversity: is habitat heterogeneity the key?", Trends in Ecology and Evolution, no 18, pp. 182-188.

Bertrand G. (1975): "Pour une histoire écologique de la France rurale", en Duby, G. y Wallon, A. (dirs.), Histoire de la France rurale, vol. 1, pp. 35-116.

Bolós, M. (dir.) (1992): Manual de ciencia del paisaje: Teoría, métodos y aplicaciones, Masson, Barcelona.

Boyce, S.G. (1995): Landscape Forestry, John Wiley and Sons Inc., Nueva York.

Bruinderink, G. G.; Van Der Sluis, T.; Lammertsma, D.; Opdam, P. y Pouwels, R. (2003): "Designing a coherent ecological network for large mammals in northwestern Europe", Conservation Biology, ${ }^{\circ}$ 17, pp. 549-557.

Burel, F. y Baudry, J. (1999): Écologie du paysage. Concepts, méthodes et applications, TEC \& DOC, París.

Burel, F. y Baudry, J. (2005): "Habitat quality and connectivity in agricultural landscapes: the role of land use systems at various scales in space and time", Ecological Indicators, $\mathrm{n}^{\circ} 5$, pp. 305-313. 
Corsi, F.; Boitani, L. y Sinibaldi. I. (2002): "Ecological corridors and species: large carnivores in the Alpine region", Nature and Environment, $n^{\circ} 127$, Council of Europe Publishing, Estrasburgo.

De Lucio, J. V.; Atauri, J. A.; Sastre, P. y Martínez, C. (2003): "Conectividad y redes de espacios naturales protegidos: del modelo teórico a la visión práctica de la gestión", García Mora, M. R. (coord.), Conectividad ambiental: las áreas protegidas en la cuenca mediterránea, Junta de Andalucía, Sevilla, pp. 29-54.

Del Barrio, G.; Simón, J. C.; Cuadrado, A.; Sánchez, E.; Ruiz-González, E. y García, R. (2000): "Aproximación para estimar la conectividad regional de las redes de conservación", en V Congreso Nacional de Medio Ambiente, Madrid, pp. 1-17.

Del Barrio, G.; Harrison, P. A.; Berry, P. M.; Butt, N.; Sanjuán, M. E.; Pearson, R. G. y Dawson, T. (2006): "Integrating multiple modelling approaches to predict the potential impacts of climate change on species' distributions in contrasting regions: comparison and implications for policy", Environmental Science and Policy, no 9, pp. 129-147.

Díaz Pineda, F. y Schmitz, M. F. (2003): "Tramas espaciales del paisaje. Conceptos, aplicabilidad y temas urgentes para la planificación territorial", en García Mora, M. R. (coord.), Conectividad ambiental: las áreas protegidas en la cuenca mediterránea, Junta de Andalucía, Sevilla, pp. 9-28.

Donald, P. F. (2004): "Biodiversity impacts of some agricultural commodity production systems", Conservation Biology, $\mathrm{n}^{\circ}$ 18, pp. 17-37.

Donald, P. F. y Evans, A. D. (2006): "Habitat connectivity and matrix restoration: the wider implications of agri-environment schemes", Journal of Applied Ecology, n 43, pp. 209-218.

Dramstad, W. E.; Olson, J. D. y Forman, R. T. T. (1996): Landscape ecology principles in landscape architecture and land-use planning, Island Press and American Society of Landscape Architects, Washington.

Europarc-España (2002): Plan de Acción para los espacios naturales protegidos del Estado español, Fundación González Bernáldez, Madrid.

Fahrig, L. (2003): "Effects of habitat fragmentation on biodiversity", Annual Review of Ecology, Evolution and Systematics, $\mathrm{n}^{\circ} 34, \mathrm{pp} .487-515$.

Farina, A. (2004): "Landscape structure and breeding bird distribution in a sub-Mediterranean agroecosystem", Landscape Ecology, n 12, pp. 365-378.

Fischer, J. y Lindenmayer, D. B. (2007): "Landscape modification and habitat fragmentation: a synthesis", Global Ecology and Biogeography, no 16, pp. 265-280.

Foley, J. A.; DeFries, R.; Asner, G. P.; Barford, C.; Bonan, G.; Carpenter, S. R.; Chapin, F. S.; Coe, M. T.; Daily, G. C.; Gibbs, H. K.; Helkowski, J. H.; Holloway, T.; Howard, E. A.; Kucharik, C. J.; Monfreda, C.; Patz, J. A.; Prentice, I. C.; Ramankutty, N. y Snyder, P. K. (2005): "Global consequences of land use", Science, no 309, pp. 570-574.

Foppen, R. P. B.; Bouwma, I. M.; Kalkhoven, J. T. R.; Dirksen, J. y Van Opstal, S. (2000): Corridors of the Pan-European Ecological Network: concepts and examples for terrestial and freshwater vertebrates, ECNC, Tilburg.

Forman, R. T. T. y Godron, M. (1981): "Patches and structural components for a Landscape Ecology", Bioscience, $\mathrm{n}^{\circ}$ 31, pp. 733-739.

Forman, R. T. T. y Godron, M. (1986): Landscape Ecology, John Wiley and Sons, Nueva York.

Forman, R. T. T. (1995): Land mosaics. The ecology of landscapes and regions, Cambridge University Press, Cambridge.

García Fernández-Velilla, S. (2003): "Conectividad en sistemas regionales de áreas protegidas", en García Mora, M. R. (coord.), Conectividad ambiental: las áreas protegidas en la cuenca mediterránea, Junta de Andalucía, Sevilla, pp. 89-110.

Gómez Orea, D. (2002): Ordenación territorial, Mundi-Prensa y Editorial Agrícola Española, Madrid.

González Bernáldez, F. (1991): "Ecological consequences of the abandonment of traditional land use systems in central Spain", en Baudry, J. y Bunce, R. G. H. (eds.), Land 
abandonment and its role in conservation, Options Méditerranéennes, Série Séminaires Méditerranéens, $\mathrm{n}^{\circ}$ 15, pp. 23-29.

Graves, T.; Farley, S.; Goldstein, M. y Servheen, C. (2007): "Identification of functional corridors with movement characteristics of brown bears on the Kenai Peninsula, Alaska", Landscape Ecology, $\mathrm{n}^{\circ} 22$, pp. 765-772.

Gurrutxaga, M. (2007): La conectividad de redes de conservación en la planificación territorial con base ecológica. Fundamentos y aplicaciones en la Comunidad Autónoma del País Vasco, Tesis doctoral, Universidad del País Vasco.

Hall, L. S.; Krausman, P. R. y Morrison, M. L. (1997): "The habitat concept and a plea for standard terminology", Wildlife Society Bulletin, ${ }^{\circ}$ 25, pp. 173-182.

Hannah, L.; Midgley, G. F. y Millar, D. (2002): "Climate change-integrated conservation strategies", Global Ecology and Biogeography, n 11, pp. 485-495.

Hoctor, T. S.; Carr, M. H. y Zwick, P. D. (2000): "Identifying a linked reserve system using a regional landscape approach: the Florida ecological network", Conservation Biology, ${ }^{\circ}$ 14, pp. 984-1000.

Huber, P. R.; Roth, N. E.; Beardsley, K.; Thorne, J. H.; McCoy, M. C. y Meade, R. (2007): "Potential impacts of urban growth on an ecological network in the San Joaquin Valley", Association for American Geographer's 2007 Annual Meeting, San Francisco, California.

Irastorza, P. (2006): Integración de la Ecología del Paisaje en la planificación territorial. Aplicación a la Comunidad de Madrid, Tesis doctoral, Universidad Politécnica de Madrid.

J’drzejewski, W.; Nowak, S.; Stachura, K.; Skierczyfski, M.; MysПajek, R.; NiedziaПkowski, K.; J’drzejewska, B.; Wójcik; J.; Zalewska, H. y Pilot, M. (2005): Project of ecological corridors linking Natura 2000 sites in Poland, Mammals Research Institute, Polish Academy of Science, Bialowieza.

Jongman, R. H. G. (2002): "Homogenisation and fragmentation of the European landscape: ecological consequences and solutions", Landscape and Urban Planning, ${ }^{\circ}$ 58, pp. 211 221.

Kent, M. (2007): "Biogeography and Landscape Ecology", Progress in Physical Geography, nº 31, pp. 345-355.

Kupfer, J. A. (1995): "Landscape Ecology and Biogeography", Progress in Physical Geography, $\mathrm{n}^{\circ} 19$, pp. 18-34.

Lambeck, R. J. (1997): "Focal species: a multi-species umbrella for nature conservation", Conservation Biology, $\mathrm{n}^{\circ} 11$, pp. 849-856.

Levins, R. (1970): "Extinction", en Gerstenhaber, M. (ed.), Some mathematical problems in biology, American Mathematical Society, Providence, pp. 75-107.

Maes, D. y Van Dyck, H. (2001): "Butterfly diversity loss in Flanders (north Belgium): Europe's worst case scenario?", Biological Conservation, no 99, 263-276.

Manel, S.; Schwartz, M. K.; Luikart, G. y Taberlet, P. (2003): "Landscape genetics: combining Landscape Ecology and population genetics", Trends in Ecology and Evolution, $\mathrm{n}^{\circ}$ 18, pp. 189-197.

Martínez de Pisón (1983): "Cultura y ciencia del paisaje", Agricultura y Sociedad, n²7, pp. 9-32.

Mata, R. (2005): Integración de los espacios naturales protegidos en la ordenación del territorio, Fundación Fernando González Bernáldez, Madrid.

Merriam, G. (1984): "Connectivity: a fundamental ecological characteristic of landscape pattern", en Brandt, J. y Agger, P., Proceedings of the First International Seminar on Methodology in Landscape Ecological Research and Planning, Roskilde Universitetsforlag GeoRuc, Roskilde, Dinamarca, pp. 5-15.

Ministerio de Medio Ambiente (1999): Estrategia para la conservación del oso pardo cantábrico, Dirección General de Conservación de la Naturaleza, Madrid.

Montes, C. (dir.) (1998): Reconocimiento biofísico de Espacios Naturales Protegidos. Doñana: una aproximación ecosistémica, Junta de Andalucía, Sevilla. 
Múgica, M.; De Lucio, J. V.; Martínez, C.; Sastre, P.; Atauri-Mezquida, J. A. y Montes, C. (2002): Integración territorial de espacios naturales protegidos y conectividad ecológica en paisajes mediterráneos, Junta de Andalucía, Sevilla.

Noss, R. F. (1991): "Landscape connectivity: different functions at different scales", en Hudson, W. E. (ed.), Landscape linkages and biodiversity, Defenders of Wildlife and Island Press, Washington, D.C.

Noss, R. F. y Daly, K. M. (2006): "Incorporating connectivity into broad-scale conservation planning", en Crooks, K. y Sanjayan, M. (eds.), Connectivity Conservation, Cambridge University Press, Cambridge, pp. 587-619.

Opdam, P.; Foppen, R. y Vos, C. (2002): "Bridging the gap between ecology and spatial planning in landscape", Landscape Ecology, n' 16, pp. 767-779.

Opdam, P.; Steingröver, E. G. y Van Rooij, S. (2006): "Ecological networks: A spatial concept for multi-actor planning of sustainable landscapes", Landscape and Urban Planning, $\mathrm{n}^{\circ}$ 75, pp. 322-332.

Pierce, A. R. y Ervin, J. B. (1999): “La certificación independiente de la ordenación forestal y la Ecología del Paisaje", Unasylva, n 50, pp. 49-56.

Pickett, S. T. A. y Cadenasso, M. L. (1995): "Landscape Ecology: Spatial heterogeneity in ecological systems", Science, $\mathrm{n}^{\circ} 269$, pp. 331-334.

Pino, J.; Rodà, F.; Ribas, J. y Pons, X. (2000): "Landscape structure and bird species richness: implications for conservation in rural areas between natural parks", Landscape and Urban Planning, $\mathrm{n}^{\circ}$ 9, pp. 5-48.

Pujadas, R. y Font, J. (1998): Ordenación y planificación territorial, Editorial Síntesis, Madrid.

Ramos Fernández, A. (ed.) (1979): Planificación física y ecología. Modelos y métodos, E.M.E.S.A., Madrid.

Ranius, T y Hedin, J. (2001): "The dispersal rate of a beetle, Osmoderma eremita, living in tree hollows", Oecologia, n 126, pp. 363-370.

Romero, M. (2005): Cambios en la estructura del paisaje del Alt Empordà en el periodo 19572001, Tesis doctoral, Universidad de Girona.

Rosenberg, D. K.; Noon, B. R. y Meslow, E. C. (1997): “Biological corridors: form, function and efficacy", Bioscience, $\mathrm{n}^{\circ} 47$, pp. 677-687.

Ryszkowski, L. (ed.). (2002): Landscape Ecology in agroecosystems management, CRC Press, Florida.

Santos, T. y Tellería, J. L. (1998): Efectos de la fragmentación de los bosques sobre los vertebrados en las mesetas ibéricas, Organismo Autónomo Parques Nacionales, Ministerio de Medio Ambiente, Madrid.

Saunders, D. A.; Hobbs, R. J. y Margules, C. R. (1991): "Biological consecuences of ecosistem fragmentation: a review", Conservation Biology, n ${ }^{\circ}$ 5, pp. 18-32.

Schafer, C. L. (1990): Nature reserves: Island theory and conservation practice, Smithsonian Institute Press, Washington.

Shaffer, M. L. (1987): "Minimum viable population: coping with uncertainty", en Soulé, M. (ed.), Viable population for conservation, Cambridge University Press, Cambridge.

Simpson, G. G. (1936): "Data on the relationships of local and continental mammalian faunas", Journal of Paleontology, n' 10, pp. 410-414.

Singleton, P. H.; Gaines, W. L. y Lehmkuhl, J. F. (2002): Landscape permeability for large carnivores in Washington: a geographic information system weighted-distance and least-cost corridor assessment, US Department of Agriculture, Forest Service, Pacific Northwest Research Station.

Suárez-Seoane, S. y Baudry, J. (2002): "Scale dependence of spatial patterns and cartography on the detection of landscape change: relationships with species' perception", ECOgraphy, $\mathrm{n}^{\circ} 25$, pp. 499-511.

Suárez-Seoane, S.; Osborne, P. E y Baudry, J. (2002): "Responses of birds of different biogeographic origins and habitats to agricultural land abandonment in northern Spain", Biological Conservation, nº 105, pp. 333-344. 
Tansley, A. (1935): "The use and abuse of vegetational concepts and terms", Ecology, n ${ }^{\circ} 16$, pp. 284-307.

Taylor, P. D.; Fahrig, L.; Henein, K. y Merriam, G. (1993): "Connectivity is a vital element of landscape structure", Oikos, n 68, pp. 571-573.

Tischendorf, L. y Fahrig, L. (2000): "How should we measure landscape connectivity?", Landscape Ecology, ${ }^{\circ} 15$, pp. 633-641.

Troll, C. (1939): "Luftbildplan und ökologische bodenforschung", Zeitschrift der gesellschaft für erdkunde zu Berlin, n 7-8, pp. 241-298.

Tucker, G. M. (1997): "Priorities for bird conservation in Europe: the importance of the farmed landscape", en Pain, D. J. y Pienkowski, M. W. (eds.), Farming and Birds in Europe: The Common Agricultural Policy and its Implications for Bird Conservation, Academic Press, London, pp. 79-116.

Turner, M. G.; O'Neill, R. V.; Garner, R. H. y Milne, B. T. (1989): "Effects of changing spatial scale on the analysis of landscape pattern", Landscape Ecology, n 3, pp. 153-162.

Turner, M. G.; Gardner, R. H y O'neill, R. V. (2001): Landscape Ecology in theory and practice, Springer-Verlag, New York.

Van Der Sluis, T.; Bloemmen, M. y Bouwma, I. M. (2004): European corridors: Strategies for corridor development for target species, ECNC, Tilburg.

Vandewoestijne, S. y Baguette, M. (2004): "Demographic versus genetic dispersal measures", Population Ecology, n 46, pp. 281-285.

Vila, J.; Varga, D.; Llausàs, A. y Ribas, A. (2006): "Conceptos y métodos fundamentales en Ecología del Paisaje (Landscape Ecology). Una interpretación desde la geografía", Documents d'Anàlisi Geogràfica, no 48, pp. 151-166.

Vos, C. C.; Verboom, J.; Opdam, P. F. M. y Ter Braak, C. F. J. (2001): "Toward ecologically scaled landscape indices", American Naturalist, n 157, pp. 24-41.

Vuilleumier, S. y Prelaz-Droux, R. (2002): “Map of ecological networks for landscape planning", Landscape and Urban Planning, $\mathrm{n}^{\circ}$ 58, pp. 157-170.

Walker, R. y Craighead, L. (1997): "Analysing wildlife movement corridors in Montana using GIS", 1997 ESRI User Conference, San Diego.

Williams, C. B. (1964): Patterns in the balance of nature and related problems in quantitative biology, New York Academy Press, Nueva York.

With, K. A. y Crist, T. O. (1995): “Critical thresholds in species' responses to landscape structure", Ecology, no 76, pp. 2446-2459.

Yanes, M.; Velasco J. M. y Suárez, F. (1995): "Permeability of roads and railways to vertebrates: the importance of culverts", Biological Conservation, $n^{\circ} 71$, pp. 217-222.

Zonneveld, I. S. (1995): Land Ecology, SPB Academic Publishing, Ámsterdam.

\section{Resumen}

Se realiza una revisión sobre las bases conceptuales y los objetivos de la Ecología del Paisaje, disciplina científica que presenta un importante componente geográfico. Como punto de partida asume que la heterogeneidad espacio-temporal del paisaje, resultante de la interacción dinámica de las sociedades humanas con el medio, controla diversos movimientos y flujos de organismos, materia y energía. Uno de los principales objetos de estudio de dicha disciplina son las relaciones existentes entre los cambios estructurales del paisaje y la dinámica de poblaciones y comunidades silvestres, configurándose como un aspecto de gran aplicabilidad en las políticas de conservación de la biodiversidad y de planificación territorial con base ecológica. En relación a esta temática, se describe el carácter transdisciplinar de la Ecología del Paisaje.

Palabras clave: Ecología del Paisaje; patrones espaciales; conservación; ordenación territorial; transdisciplinariedad. 


\begin{abstract}
A review on the conceptual basis and aims of Landscape Ecology, scientific discipline that presents an important geographical component, is realized. It assumes that landscape heterogeneity controls diverse movements and flows of organisms, matter and energy. One of the principal objects of study of the above mentioned discipline is the existing relations between landscape structural patterns and dynamics of wild populations and communities. This is an aspect of great applicability in biodiversity conservation and land-use planning policies. In relation to this subject matter, transdisciplinarity of Landscape Ecology is described.
\end{abstract}

Key words: Lanscape Ecology; spatial patterns; conservation;; land-use planning; transdisciplinarity. 